\title{
Differential Role of Neutrophils and Alveolar Macrophages in Hepatocyte Growth Factor Production in Pulmonary Fibrosis
}

\author{
Bruno Crestani, Monique Dehoux, Gilles Hayem, Véronique Leçon, \\ Francine Hochedez, Joëlle Marchal, Sandrine Jaffré, Jean-Baptiste Stern, \\ Geneviève Durand, Dominique Valeyre, Michel Fournier, and Michel Aubier
}

INSERM Unit 408 (BC, MD, JM, SJ, J-BS, MF, MA), Faculté Xavier Bichat, and Laboratoire de Biochimie A (MD, VL, FH, GD), Service de Pneumologie (BC, MA), and Service de Rhumatologie (GH), Hôpital Bichat, Paris, and Service de Pneumologie (MF), Hôpital Beaujon, Clichy, and Service de Pneumologie (DV), Hôpital Avicenne, Bobigny, Assistance Publique-Hôpitaux de Paris, France

SUMMARY: Neutrophils may participate in the development of lung fibrosis. Hepatocyte growth factor (HGF), a growth factor for type II pneumocytes, is produced by neutrophils. We measured the production of HGF by blood and alveolar neutrophils from patients with either idiopathic pulmonary fibrosis $(n=11)$ or connective tissue disease-associated pulmonary fibrosis $(n=10)$ and from control patients $(n=10)$. HGF secretion by alveolar macrophages and the expression of the HGF receptor by alveolar epithelial cells in pulmonary fibrosis were also evaluated. HGF was not detected in bronchoalveolar lavage fluid from controls. HGF concentration in the epithelial lining fluid from patients was 4-fold higher than in plasma, suggesting a local production within the alveolar space. Alveolar neutrophils secreted HGF in vitro. Basal HGF secretion by alveolar neutrophils positively correlated with HGF in the epithelial lining fluid $(p=0.05$, rho $=0.582$ ). HGF secretion by alveolar neutrophils could not be further stimulated with lipopolysaccharide, whereas HGF secretion by blood neutrophils doubled with lipopolysaccharide. Alveolar macrophages did not secrete HGF in vitro. The expression of the HGF receptor was greatly increased in the fibrotic lung, supporting the local function of HGF secreted by neutrophils. We conclude that neutrophils are a source of HGF in patients with pulmonary fibrosis. (Lab Invest 2002, 82:1015-1022).

$P$ ulmonary fibrosis, either idiopathic or associated with a pathologic condition (such as a connective tissue disease), is characterized by fibroblast proliferation and extracellular matrix remodeling, which result in irreversible distortion of the lung's architecture. Chronic inflammation of the alveolar space has long been thought to mediate the development of pulmonary fibrosis, in part through alveolar epithelial injury, activation, and delayed repair, which is now considered as a critical event in lung fibrogenesis (Selman et al, 2001). Alveolar epithelial repair is a complex and finely regulated process, based upon migration and proliferation of type 2 alveolar epithelial cells (Bitterman et al, 1994). Growth factors have been shown to control both phases of the process. Among these factors, the hepatocyte growth factor (HGF), a heterodimeric protein obtained through the cleavage of an inactive precursor, proHGF, has been demon-

DOI: 10.1097/01.LAB.0000024361.92500.19

Received February 12, 2002.

This work was supported by a grant from the Programme Hospitalier de Recherche Clinique (P970714).

Address reprint requests to: Dr. Bruno Crestani, Service de Pneumologie, Hôpital Bichat, 46 rue Henri Huchard, 75877 Paris Cedex 18, France. E-mail:bruno.crestani@bch.ap-hop-paris.fr strated to increase migration (Kim et al, 1999) and proliferation (Mason et al, 1994; Ohmichi et al, 1996; Panos et al, 1996) of type 2 cells in vitro and in vivo and to limit lung fibrosis in vivo after bleomycin injury in rodents when given intravenously (Yaekashiwa et al, 1997) or intratracheally (Dohi et al, 2000). HGF acts through the MET receptor, a membrane-bound tyrosine kinase (Weidner et al, 1993). HGF has been detected in the blood and the bronchoalveolar lavage (BAL) fluid of patients with pulmonary fibrosis (Yamanouchi et al, 1998). However, the cell sources of HGF in the lung are not well known in this condition; in particular, the role of alveolar macrophages remains under debate. Moreover, the expression of the HGF receptor has not been adequately assessed in the fibrotic lung.

A neutrophilic alveolitis is very common in pulmonary fibrosis, either idiopathic (IPF) or associated with a connective tissue disease (CTD-PF) (Haslam et al, 1980). The critical role of persistent neutrophil accumulation in the airspace, leading to persistent injury of the epithelial barrier with abnormal repair, has been advocated in the genesis of interstitial fibrosis (Hunninghake et al, 1981). Part of the injurious effect of neutrophils in the lung is thought to be mediated by the local release of proteases overwhelming the local antiprotease defenses (Crestani et al, 1994). However, 
recent data suggest that neutrophils may have a beneficial effect in alveolar repair. The later could be mediated through the secretion of growth factors directed to the epithelium. Indeed, results of our laboratory indicate that blood neutrophils secrete HGF in vitro in response to different stimuli, including lipopolysaccharide (LPS) (Grenier et al, 2002), and that alveolar neutrophils could be a significant source of HGF in acute lung injury (Jaffre et al, 2002).

Therefore, the aim of this study was to (a) determine whether neutrophils participate in HGF production in the lungs of patients with IPF or CTD-PF, (b) compare alveolar macrophage and neutrophil HGF secretion in these diseases, and (c) evaluate the level of expression of the HGF receptor (c-met) by alveolar epithelial cells in the fibrotic lung.

\section{Results}

\section{Plasma and Alveolar HGF Concentrations}

HGF was detected in the plasma in both control subjects and patients. The plasma HGF concentration in patients with IPF (859 pg/ml [698-956]) and CTD-PF (763 pg/ml [650-1018]) was significantly higher than that of controls $(464 \mathrm{pg} / \mathrm{ml}$ [407-547], $p=0.0001$ versus controls).

HGF was detected in all of the BAL supernatants from patients (70 pg/ml [47-123]). HGF was below the detection level in the BAL fluid from all controls. BAL HGF concentration was not different in IPF $(70 \mathrm{pg} / \mathrm{ml}$ [54-90]) and CTD-PF (78 pg/ml [40-147], $p=0.49)$. Plasma and BAL HGF concentrations correlated positively ( $r$ o $=0.476, p=0.03$ ).

To compare lung and plasma HGF levels, we calculated the HGF concentration in the epithelial lining fluid (ELF). HGF concentration in ELF was similar in IPF (3278 pg/ml [2127-9831]) and CTD-PF (3460 $\mathrm{pg} / \mathrm{ml}$ [2342-13345]) and was 4-fold higher than in plasma $(p=0.009$ and $p=0.01$ for IPF and CTD-PF, respectively), thus indicating some intra-alveolar production of HGF. BAL HGF tended to correlate with the percentage of neutrophils in $\mathrm{BAL}$ ( $\mathrm{rho}=0.4, p=0.07$ ).

\section{HGF Secretion by Alveolar Neutrophils}

Alveolar neutrophils from patients stained strongly positive with an anti-HGF antibody (Fig. 1). We evaluated the ability of alveolar neutrophils to secrete HGF in vitro. Basal HGF secretion could be measured for 11 patients. Alveolar neutrophils from patients with IPF and CTD-PF released similar levels of HGF in vitro (542 pg/ml/10 ${ }^{7}$ neutrophils [143-800], $n=6$, and 510 $\mathrm{pg} / \mathrm{ml} / 10^{7}$ neutrophils [367-658], $n=5$, respectively) (Fig. 2).

The limited number of BAL neutrophils allowed us to evaluate LPS-induced HGF secretion in only five patients (IPF, $n=4$; CTD-PF, $n=1$ ). LPS did not increase the HGF release by alveolar neutrophils in comparison with basal secretion $\left(571 \mathrm{pg} / \mathrm{ml} / 10^{7}\right.$ neutrophils [128-1025] versus $535 \mathrm{pg} / \mathrm{ml} / 10^{7}$ neutrophils [120-984], respectively, $p=0.27$ versus basal secretion).
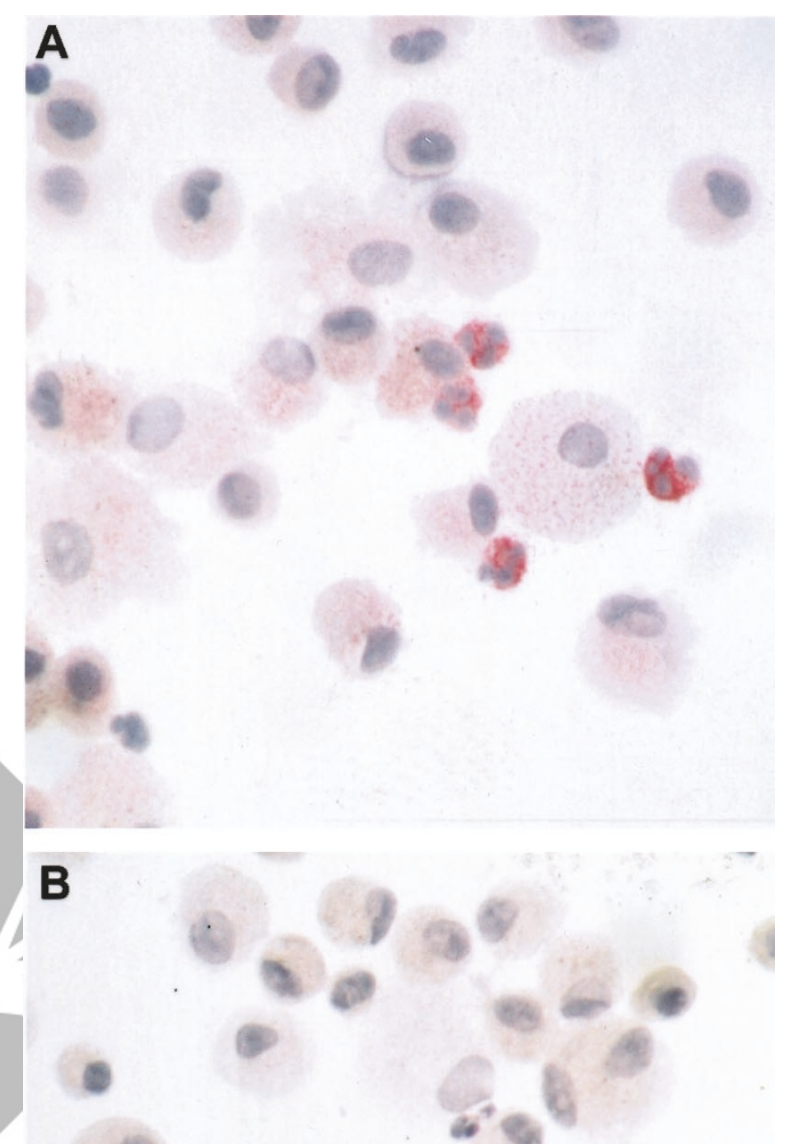

1

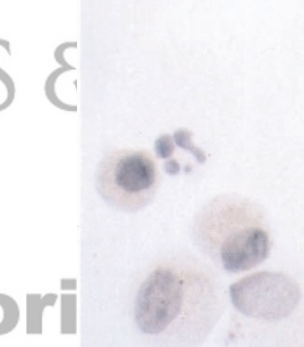

\section{Figure 1.}

Hepatocyte growth factor (HGF) immunostaining of bronchoalveolar lavage (BAL) cells. BAL cytocentrifuge smears were immunostained with a monoclonal anti-human-HGF antibody or with a nonspecific mouse immunoglobulin of the same isotype as a negative control (magnification, $\times 800$ ). A, Alveolar neutrophils stained strongly positive, whereas macrophages were weakly positive. B, Control antibody.

Spontaneous HGF release by alveolar neutrophils positively correlated with HGF concentration in ELF (rho $=0.582, p=0.05$ ) when the two groups of fibrotic patients were taken as a whole. HGF release by alveolar neutrophils did not correlate with any of the lung function tests or blood gas results.

\section{Lack of HGF Secretion by Alveolar Macrophages}

Alveolar macrophages were weakly stained with an anti-HGF antibody (Fig. 1). HGF was always below the 


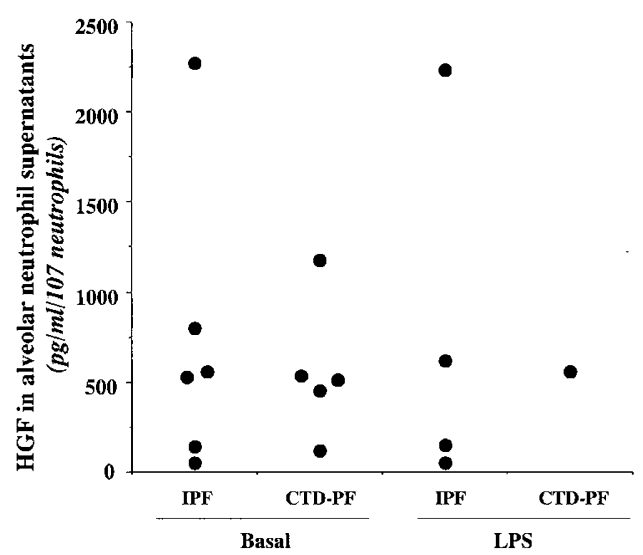

Figure 2.

HGF concentration in BAL neutrophils culture supernatants from patients with idiopathic pulmonary fibrosis (IPF) and connective tissue disease-associated pulmonary fibrosis (CTD-PF). Individual values. Neutrophils were purified and cultured for 20 hours $\left(10^{7} / \mathrm{ml}\right.$ per well) in basal conditions or with LPS stimulation $(1 \mu \mathrm{g} / \mathrm{ml})(L P S)$. HGF concentrations were not different in each group.

detection level of the ELISA in alveolar macrophage supernatants, either basal or LPS stimulated, obtained from seven IPF patients and from eight control subjects.

\section{HGF Secretion by Blood Neutrophils}

Basal HGF secretion by blood neutrophils was higher in patients with CTD-PF $\left(1136 \mathrm{pg} / \mathrm{ml} / 10^{7}\right.$ neutrophils [941-1607], $n=10)$ than in patients with IPF (511 $\mathrm{pg} / \mathrm{ml} / 10^{7}$ neutrophils [435-968], $n=9, p=0.04$ ) and in controls $\left(823 \mathrm{pg} / \mathrm{ml} / 10^{7}\right.$ neutrophils [643-949], $p=$ 0.05) (Fig. 3).

Although alveolar neutrophils could not be stimulated with LPS, HGF secretion by blood neutrophils could be increased 2-fold after LPS stimulation, either in IPF (1736 pg/ml/10 7 neutrophils [1596-1909], $n=6)$ or in CTD-PF (1862 pg/ml/10 neutrophils [1501-

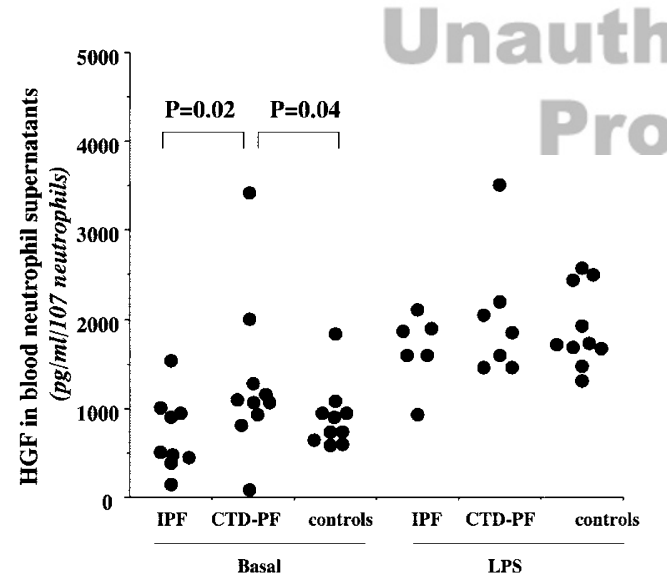

Figure 3.

HGF concentration in blood neutrophil culture supernatants from patients with IPF and CTD-PF and from controls. Individual values. Neutrophils were purified and cultured for 20 hours $\left(10^{7} / \mathrm{ml}\right.$ per well) in basal conditions or with lipopolysaccharide stimulation $(1 \mu \mathrm{g} / \mathrm{ml})$ (LPS). HGF secretion was higher in CTD-PF than in IPF $(p=0.02)$ or in controls $(p=0.04)$. LPS stimulation increased HGF production in each group.
2166], $n=7)$ or in controls $\left(1730 \mathrm{pg} / \mathrm{ml} / 10^{7}\right.$ neutrophils [1682-2445]). LPS-stimulated HGF secretion was similar in the three groups. For each patient, HGF release by blood neutrophils was significantly higher than that from alveolar neutrophils, either unstimulated $(p=0.01)$ or LPS stimulated $(p=0.05)$. Basal HGF secretion by blood neutrophils from patients correlated positively with forced vital capacity (rho $=$ $0.455, p=0.05$ ), forced expiratory volume in one second (rho $=0.486, p=0.04$ ), and BAL albumin (rho $=0.584, p=0.01$ ) but did not correlate with total lung capacity, carbon monoxide diffusion capacity, or arterial blood gases.

\section{HGF Receptor Is Strongly Expressed in the Fibrotic Lung}

To evaluate the biologic significance of our data, we asked whether HGF receptor was expressed in the fibrotic lung. HGF receptor expression has been shown to be reduced in acute lung injury (Yanagita et al, 1993) but has not been evaluated in chronic lung injury. Immunohistochemistry demonstrated some expression of c-met on endothelial cells and alveolar epithelial cells in the normal lung, whereas alveolar macrophages stained negative (Fig. 4C). We studied lung samples from three different patients with endstage pulmonary fibrosis who were undergoing transplantation. In all fibrotic lung samples, HGF receptor expression was greatly increased, especially on hyperplastic alveolar epithelial cells (Fig. 4A). Some positive cells, probably desquamated epithelial cells, were detected in the airspaces. Endothelial cells were still positively stained (Fig. 4).

\section{Discussion}

The alveolar epithelium plays a critical role in the complex repair process that follows lung injury and has been implicated in the pathophysiology of lung fibrosis (Selman et al, 2001). HGF, a multipotent growth factor with mitogenic, motogenic, and morphogenic effects, stimulates the proliferative and repair capacity of the alveolar epithelium (Dohi et al, 2000; Ware and Matthay, 2002; Yaekashiwa et al, 1997). Our results confirmed the production of HGF in the lung of patients with pulmonary fibrosis and demonstrated that blood and alveolar neutrophils are a significant source of HGF as opposed to alveolar macrophages. Moreover, we showed that the expression of the HGF receptor is increased in the alveolar space in patients with pulmonary fibrosis, in comparison with the normal lung.

Our results indicate local production of HGF in the lungs of patients with pulmonary fibrosis because HGF concentration in the ELF was higher than in plasma. HGF production in the lung has been previously evidenced in patients with acute lung injury (Stern et al, 2000; Verghese et al, 1998) and in patients with pulmonary fibrosis (Yamanouchi et al, 1998). However, the nature of the HGF-producing cells in these pathologic situations remains to be determined. Fibroblasts, endothelial cells, and perhaps hyperplas- 


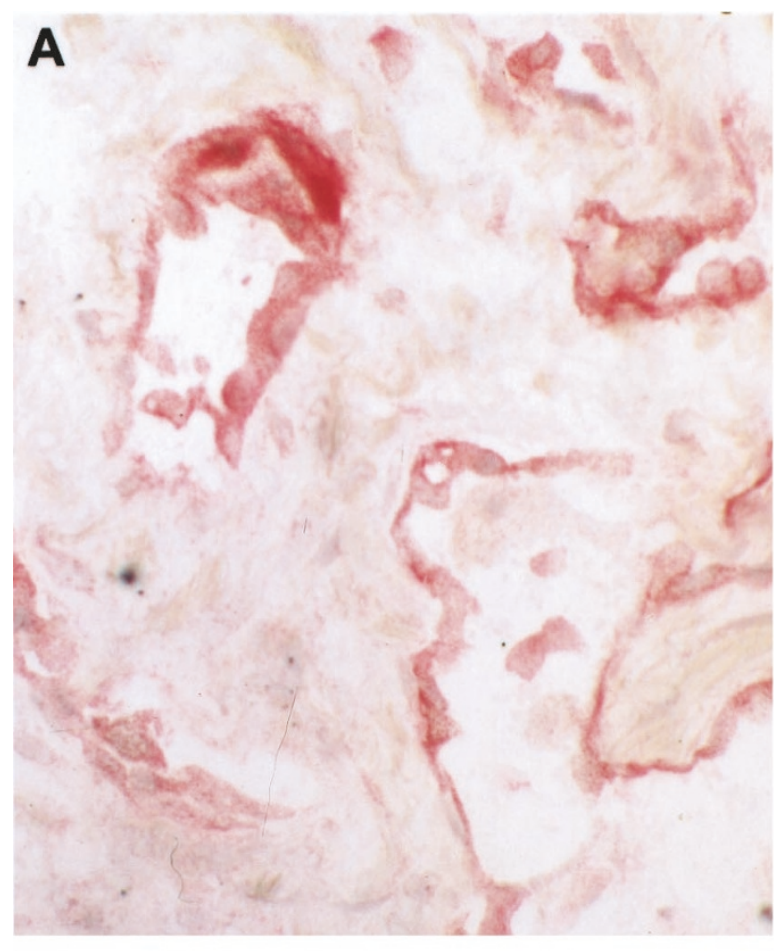

B

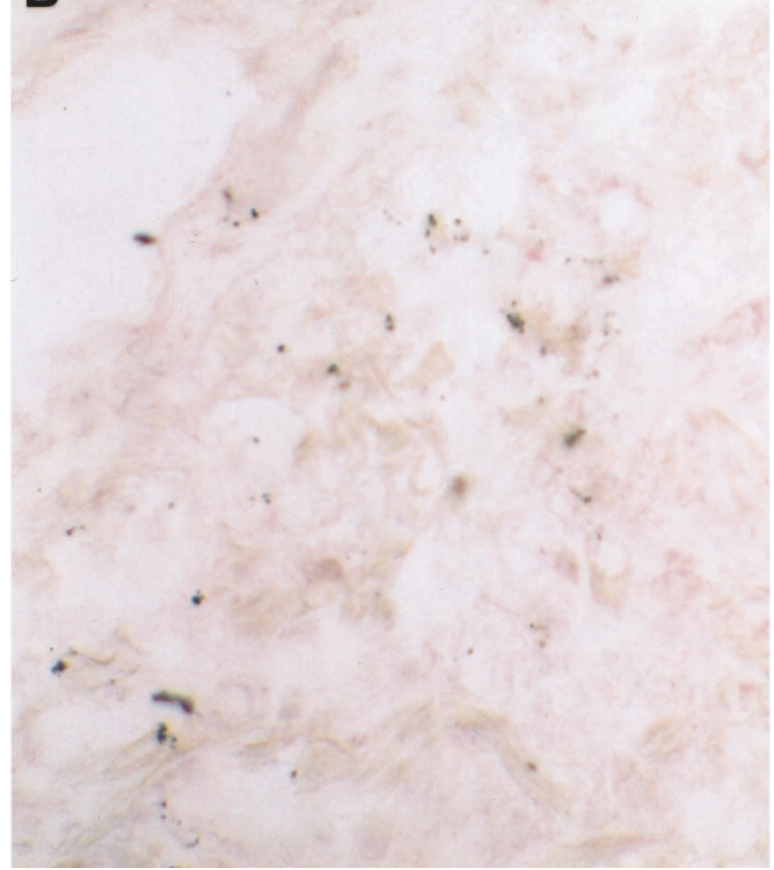

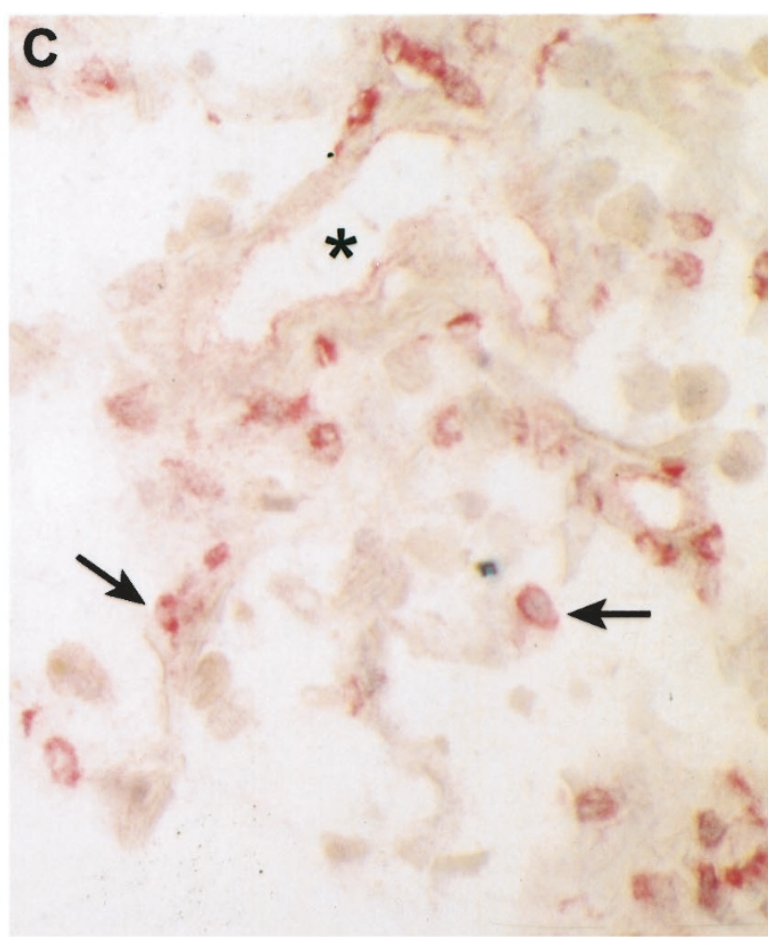

D

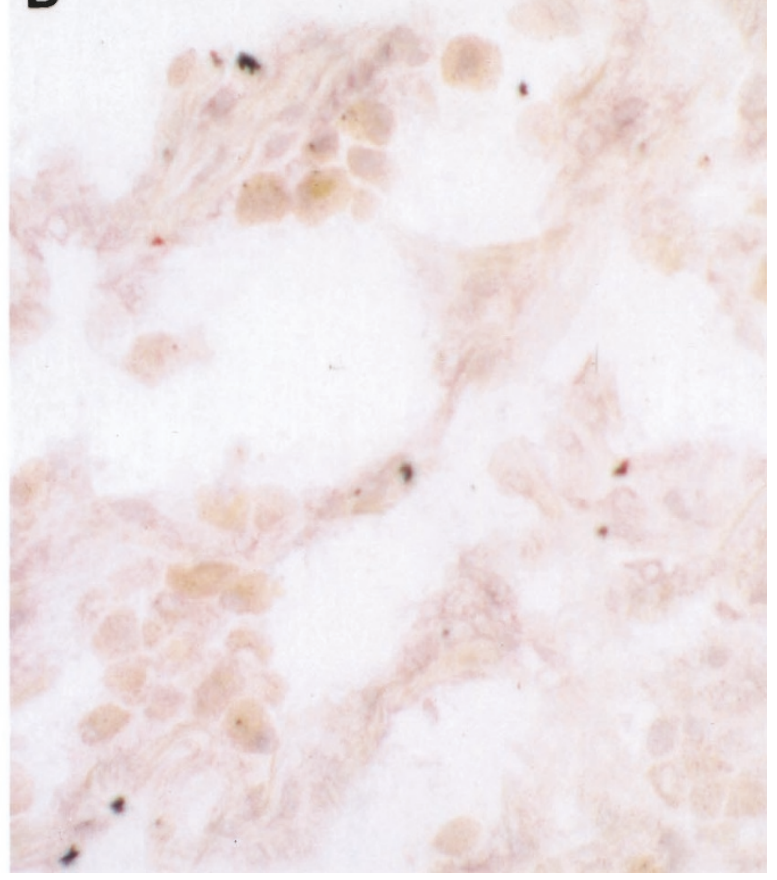

\section{Figure 4.}

Immunostaining for HGF receptor in the lung. The hyperplastic alveolar epithelium strongly expressed the HGF receptor in the fibrotic lung (A). In the normal lung $(\mathrm{C})$, staining was limited to type 2 pneumocytes (arrows) and endothelial cells in the vascular lumen (asterisk). The control antibody gave no staining (B and D).

tic alveolar epithelial cells are a source of HGF in the human lung (Sakai et al, 1997). Our data suggest that blood and alveolar neutrophils significantly contribute to the total alveolar burden of HGF in pulmonary fibrosis. Immunocytochemical analysis demonstrated that alveolar neutrophils contained HGF. Furthermore, we observed that blood and BAL neutrophils released HGF in vitro, that HGF release by alveolar neutrophils correlated positively with HGF concentration in ELF, and that HGF concentration in BAL supernatant tended to correlate with the percentage of neutrophils in BAL. We have previously observed that HGF concentration in BAL supernatant positively correlated with the percentage of neutrophils in BAL in two different groups of patients with acute lung injury (Jaffre et al, 2002; Stern et al, 2000). Altogether, our 
results indicate that neutrophils contribute to alveolar HGF production both in acute and chronic lung injury, although we cannot determine quantitatively how important neutrophils are as a source of HGF in patients with pulmonary fibrosis. Reciprocally, HGF could also stimulate the migration of neutrophils to the alveolar space. In vitro, HGF induces the lymphocyte functionassociated antigen-1-mediated adhesion of neutrophils to endothelial cells and stimulates the transmigration of neutrophils in a concentration-dependent manner (Mine et al, 1998).

Our data suggest that alveolar macrophages are not a significant source of HGF in the lung. Indeed, although alveolar macrophages stained weakly positive with an anti-HGF antibody (Fig. 1), we were unable to detect any HGF secretion in vitro by alveolar macrophages, from controls and from IPF patients, either unstimulated or stimulated with LPS. Furthermore, in four experiments, we studied HGF secretion by alveolar macrophages stimulated with either $10^{-6}$ $\mathrm{M}$ phorbol myristate acetate (PMA) or $10^{-3} \mathrm{M}$ dibutyryl cyclic AMP. In both conditions, HGF was not detected in macrophage supernatants (unpublished data). HGF was not detected in PMA-stimulated alveolar macrophage supernatants. Lack of HGF secretion by human alveolar macrophages is further supported by Mason and colleagues (Mason et al, 1994) who previously showed that human alveolar macrophages do not contain HGF mRNA, whereas rat alveolar macrophages do. Sakai and colleagues (Sakai et al, 1997) found that human alveolar macrophages contained immunoreactive HGF, but they did not study HGF secretion by alveolar macrophages. LPS-stimulated human blood monocytes have been shown to secrete small amounts of HGF in vitro, whereas unstimulated monocytes do not (Galimi et/al, 2001).

It is worth noting that spontaneous HGF secretion by blood neutrophils was 2-fold higher in patients with CTD-PF than in patients with IPF. Neutrophils are known to be activated in patients with systemic sclerosis (Maslen et al, 1987), but we are not aware of a direct comparison of blood neutrophils from IPF and CTD-PF. Three patients with CTD-PF received corticosteroids at the time of examination, but a stimulatory effect of corticosteroids is unlikely because corticosteroids inhibit HGF production in human lung fibroblasts and leukemic cells in vitro (Matsumoto et al, 1992).

Basal HGF secretion by alveolar neutrophils was lower than basal HGF secretion by blood neutrophils and was not increased by LPS stimulation. We obtained similar results when we studied HGF secretion by alveolar neutrophils obtained from ventilated patients with acute lung injury (Jaffre et al, 2002). We hypothesize that the lack of response to LPS is a result of the degranulation of neutrophils when migrating to the alveolar space as previously suggested by others (Cailes et al, 1996). Further studies are needed to confirm this hypothesis.

The concentration of HGF in ELF in our study was about $3 \mathrm{ng} / \mathrm{ml}$ in patients. This concentration is biologically significant because similar levels of HGF increase $\left[{ }^{3} \mathrm{H}\right]$ thymidine incorporation by type II pneumocytes in vitro (Mason et al, 1994). Moreover, we demonstrate that the expression of the HGF receptor was increased in patients with pulmonary fibrosis, particularly on hyperplastic alveolar epithelium. Endothelial cells stained positive as already known. This is important information that contrasts with the acute phase of lung injury in which the c-met expression was shown to be down-regulated in the lung (Yanagita et al, 1993). Inflammatory cytokines probably participate in the strong expression of the HGF receptor in the fibrotic lung. Inflammatory cytokines present in the fibrotic lung (such as IL- $1 \alpha$, TNF- $\alpha$, IL-6, transforming growth factor- $\beta 1$, epidermal growth factor, and HGF) up-regulate the c-met expression in several types of carcinoma cell lines (Moghul et al, 1994). Moreover, IFN- $\gamma$ up-regulates the expression of c-met in vitro in A549 epithelial cells and in vivo in the lung in mice (Nagahori et al, 1999). Altogether, these data support a biologic role for HGF secretion by neutrophils in the alveolar repair phenomenon in the fibrotic lung.

The chronic infiltration of alveolar structures by neutrophils may release HGF in the immediate vicinity of its main targets, epithelial and endothelial cells. In different animal models of acute lung injury, intratracheal or intravenous HGF administration over a short period of time decreases the extent of the pulmonary lesions and improves the survival of the animals (Ohmichi et al, 1996; Panos et al, 1996; Yaekashiwa et al, 1997) even when given after the insult. HGF may exert its protective action through different pathways: stimulation of the proliferation (Mason et al, 1994) and migration (Kim et al, 1999) of type II pneumocytes, inhibition of their apoptosis (Fan et al, 2000), and stimulation of angiogenesis (Aoki et al, 2000). Will chronically elevated HGF concentrations be beneficial? One may speculate that HGF may have local deleterious effects. HGF inhibits the synthesis and secretion of the pulmonary surfactant phospholipids (Vivekananda et al, 2000). HGF may induce the development of a hyperplastic alveolar epithelium, a very common abnormality in pulmonary fibrosis (Kawanami et al, 1982), not adapted to gas exchange. Finally, chronic HGF increase may contribute to the high incidence of lung cancer in patients with pulmonary fibrosis, particularly of the bronchiolo-alveolar type (Hubbard et al, 2000; Yamashita et al, 1998).

In conclusion, our results demonstrate that blood and alveolar neutrophils contribute to the alveolar production of HGF in pulmonary fibrosis, whereas alveolar macrophages do not. Our findings provide evidence of increased HGF receptor expression in pulmonary fibrosis, essentially on hyperplastic alveolar epithelium.

\section{Materials and Methods}

\section{Patients and Controls}

The study was approved by the ethics committee of Paris-Bichat University Hospital. HGF expression was studied in three groups of subjects: 11 patients with IPF, 10 patients with CTD-PF (polymyositis, $n=$ 
5; systemic sclerosis, $n=3$; Sjögren syndrome, $n=$ 1 ; rheumatoid arthritis, $n=1$ ), and 10 control patients (4 current smokers). Controls underwent a fiberoptic bronchoscopy for the diagnostic evaluation of an esophageal cancer $(n=4)$ or a lung nodule $(n=3)$ and for suspected hemoptysis $(n=$ 3). In all control patients, the fiberoptic bronchoscopy was normal, and the BAL was performed in a radiographically normal area. None of the controls received corticosteroids or immunosuppressant drugs. Patients and controls were free of infection at the time of examination.

All patients underwent clinical evaluation, including chest radiography, lung function measurements, and thin-section computed tomography before the fiberoptic bronchoscopy and BAL. Results of lung function tests and BAL are given in Table 1. None of the patients was an active smoker at the time of diagnosis; however, one patient with CTD-PF and four patients with IPF were former smokers. None of the patients with IPF received corticosteroids or immunosuppressants at the time of examination. Three patients with CTD-PF received prednisone at the time of examination $(10,12$, and $15 \mathrm{mg} /$ day, respectively) associated with azathioprine (125 mg/ day) in one patient. A surgical lung biopsy was not performed in any of the patients studied. In all the patients, the presence of pulmonary fibrosis was determined by clinical, functional, and computed tomography findings (bibasilar reticular abnormalities with traction bronchiectasias) analyzed by experienced pulmonologists (B.C., D.V., M.F., and M.A.). In the absence of a surgical lung biopsy, IPF was defined according to the international consensus statement (Anonymous, 2000).

\section{Blood and BAL Recovery}

BAL was performed in the right middle lobe using standard techniques (Crestani et al, 1994). The cells were separated from the fluid by centrifugation. The supernatant was frozen at $-20^{\circ} \mathrm{C}$ with $5 \%$ aprotinin (vol/vol) (Trasylol; Bayer Pharma, Sens, France) until HGF, albumin, and urea assays were performed. The cell pellet was resuspended in PBS $\left(10^{7}\right.$ cells $\left./ \mathrm{ml}\right)$ and used for neutrophil purification. A small aliquot was cytocentrifuged, air dried, and stained using May-GrünwaldGiemsa stain for a differential cell count (Table 1).

Venous blood samples $(5 \mathrm{ml})$ were obtained from patients and control subjects onto EDTA immediately before performing the bronchoscopy. One milliliter was immediately centrifuged to recover plasma, which was stored at $-20^{\circ} \mathrm{C}$ until HGF and urea assays. Four milliliters were used for neutrophil purification.

\section{Polymorphonuclear Neutrophils Preparation}

Blood and BAL neutrophils were purified and cultured as previously described (Grenier et al, 2001). Briefly, $10^{7}$ neutrophils $/ \mathrm{ml}$ were cultured in the presence or absence of LPS $(1 \mu \mathrm{g} / \mathrm{ml})$ for 20 hours in DMEM with $10 \%$ fetal bovine serum. Supernatants were then recovered and stored at $-20^{\circ} \mathrm{C}$ until HGF assay. Because neutrophils were virtually absent in BAL from control subjects and were present at a low percentage in BAL in several patients, alveolar neutrophils could be purified in a significant amount from only 11 patients with lung fibrosis (6 IPF, 5 CTD-PF).

\section{Alveolar Macrophages Preparation}

To compare HGF secretion from alveolar neutrophils and alveolar macrophages, alveolar macrophages

Table 1. Lung Function Tests and Bronchoalveolar Lavage (BAL) Results in Patients

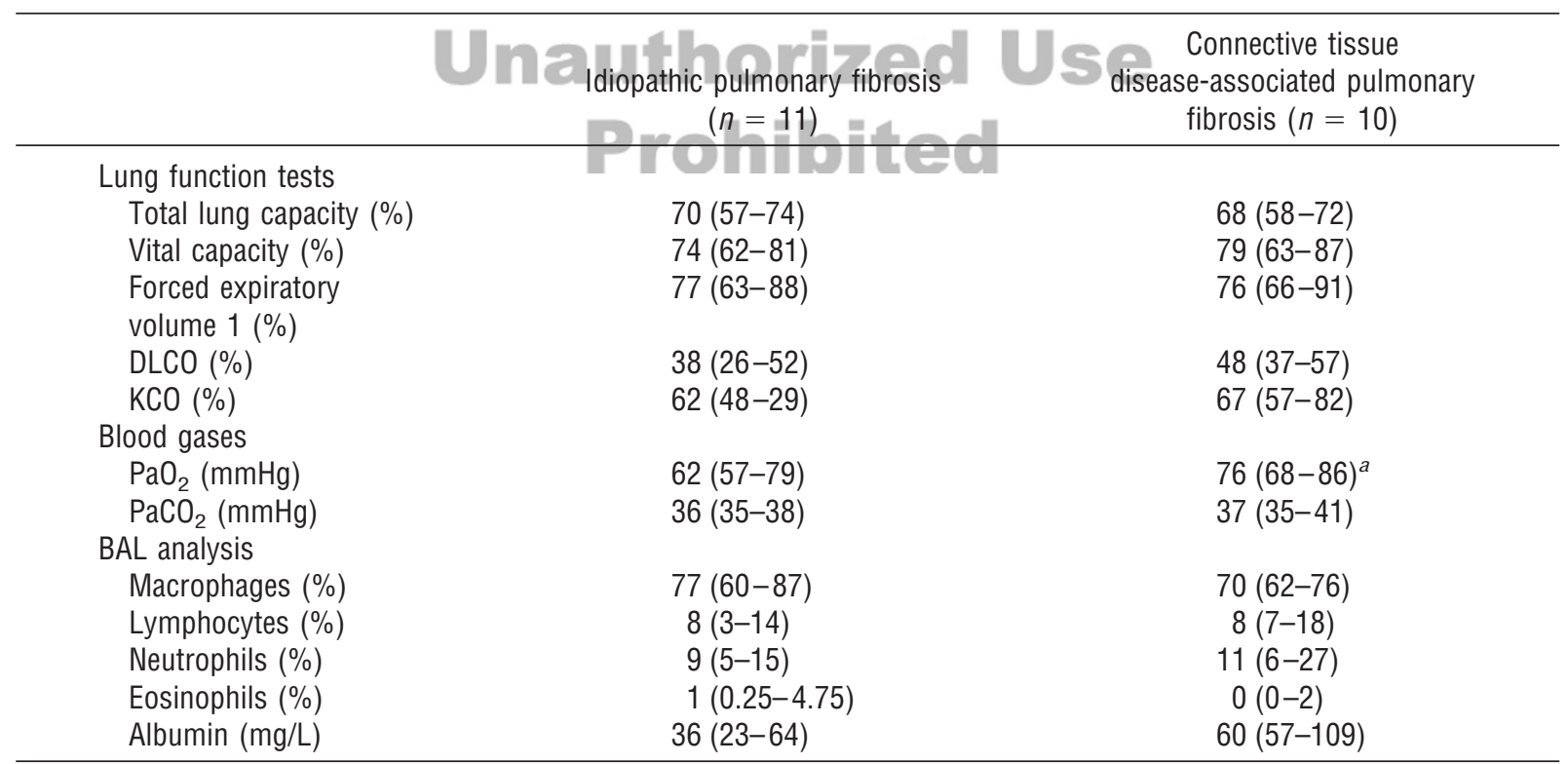

DLCO, diffusion capacity for carbon monoxide; $\mathrm{KCO}$, transfer coefficient for carbon monoxide.

${ }^{a} p<0.05$ vs idiopathic pulmonary fibrosis. Results are medians (25th to 75 th percentiles). 
from seven patients with IPF and eight controls were purified and cultured from BAL fluid as previously described (Crestani et al, 1994). Briefly, alveolar macrophages $\left(10^{6} \mathrm{cells} /\right.$ dish of a 6 -well cell culture plate) were cultured during 20 hours in fresh RPMI 1640 supplemented with $5 \%$ heat-inactivated fetal bovine serum with or without LPS $(1 \mu \mathrm{g} / \mathrm{ml})$. In four experiments, alveolar macrophages were stimulated with $10^{-6} \mathrm{M}$ PMA. Supernatants were recovered and stored at $-20^{\circ} \mathrm{C}$ until HGF assay.

\section{HGF, Albumin, and Urea Assay}

HGF was measured in plasma, BAL fluid, and neutrophil supernatants by using a commercial ELISA kit (Quantikine; R\&D Systems, Abingdon, United Kingdom). The detection limit was $40 \mathrm{pg} / \mathrm{ml}$.

BAL urea concentration was measured with a multiparametric analyst Hitachi 947 (Roche-Boehringer, Grenoble, France). BAL albumin concentration was measured with the analyst Hitachi 911 (RocheBoehringer). The concentration of HGF in the ELF was estimated according to the method of Rennard et al (1986) by the following formula:

HGFELF $\left(\mathrm{pg} / \mathrm{ml}\right.$ of ELF) $=\mathrm{HGF}^{\mathrm{BAL}}$ fluid urea $^{\text {plasma }} /$ urea $^{\text {BAL fluid }}$

\section{Immunohistochemical Detection of HGF and the HGF Receptor}

Immunostaining of BAL cells was performed using anti-human-HGF monoclonal antibody (12.5 $\mu \mathrm{g} / \mathrm{ml})$ (MAB294, clone 24612.111; R\&D Systems) as previously described (Jaffre et al, 2002). A nonspecific mouse immunoglobulin of the same isotype $(10 \mu \mathrm{g} / \mathrm{ml})$ (R\&D Systems) served as a negative control.

HGF receptor (c-met) was detected on lung tissue obtained from three different patients with end-stage pulmonary fibrosis who were undergoing transplantation. Pathologic analysis was consistent with endstage usual interstitial pneumonia. Grossly normal lung tissue taken at a site distant from the tumor was obtained from three patients ( $62 \pm 4$ years, all smokers) at the time of the thoracotomy for primary lung carcinoma. Tissue fragments were immediately frozen in liquid N2 and stored at $-80^{\circ} \mathrm{C}$ until use. Cryostat sections (4- to 6-mm thick) were fixed in acetone and reacted with $7.5 \mu \mathrm{g} / \mathrm{ml}$ goat polyclonal IgG anti c-met (ref. AF726; R\&D Systems). This antibody was raised against the extracellular domain of the human HGF receptor. Control slides were incubated with $7.5 \mu \mathrm{g} / \mathrm{ml}$ control goat IgG (Vector Laboratories, Burlingame, California).

\section{Statistical Analysis}

Results are expressed as median and 25th to 75th percentiles. All assays were performed in duplicate, and the mean values were used in subsequent data analysis. Differences between the data obtained from different cultures of a given patient were analyzed with the Wilcoxon's paired nonparametric test. The significance of differences between patients and control subjects was determined with the Mann-Whitney $U$ test. Correlation between non-normally distributed variables used the Spearman's rank-order test. For statistical analysis, the concentrations of HGF below the detection limit were assigned to the value of the detection limit $(40 \mathrm{pg} / \mathrm{ml})$.

\section{References}

Anonymous (2000). Idiopathic pulmonary fibrosis: Diagnosis and treatment. International consensus statement. Am J Respir Crit Care Med 161:646-664.

Aoki M, Morishita R, Taniyama Y, Kida I, Moriguchi A, Matsumoto K, Nakamura T, Kaneda Y, Higaki J, and Ogihara $T$ (2000). Angiogenesis induced by hepatocyte growth factor in non-infarcted myocardium and infarcted myocardium: Up-regulation of essential transcription factor for angiogenesis, ets. Gene Ther 7:417-427.

Bitterman PB, Plunovsky VA, and Ingbar DH (1994). Repair after acute lung injury. Chest 105:118S-121S.

Cailes JB, O'Connor C, Pantelidis P, Southcott AM, Fitzgerald MX, Black CM, and du Bois RM (1996). Neutrophil activation in fibrosing alveolitis: A comparison of lone cryptogenic fibrosing alveolitis and systemic sclerosis. Eur Respir J 9:992-999.

Crestani B, Seta N, Palazzo E, Rolland C, Venembre P, Dehoux M, Boutten A, Soler P, Dombret M-C, Kahn MF, and Aubier M (1994). Interleukin-8 and neutrophils in systemic sclerosis with lung involvement. Am J Respir Crit Care Med 150:1363-1367.

Dohi M, Hasegawa T, Yamamoto K, and Marshall BC (2000). Hepatocyte growth factor attenuates collagen accumulation in a murine model of pulmonary fibrosis. Am J Respir Crit Care Med 162:2302-2307.

Fan S, Ma YX, Wang JA, Yuan RQ, Meng Q, Cao Y, Laterra JJ, Goldberg ID, and Rosen EM (2000). The cytokine hepatocyte growth factor/scatter factor inhibits apoptosis and enhances DNA repair by a common mechanism involving signaling through phosphatidyl inositol 3' kinase. Oncogene 19:2212-2223.

Galimi F, Cottone E, Vigna E, Arena N, Boccaccio C, Giordano S, Naldini L, and Comoglio PM (2001). Hepatocyte growth factor is a regulator of monocyte-macrophage function. J Immunol 166:1241-1247.

Grenier A, Chollet-Martin S, Crestani B, Delarche C, El Benna J, Jaffré S, Durand G, Gougerot-Pocidalo MA, Aubier M, and Dehoux M (2002). Presence of a mobilizable intracellular pool of hepatocyte growth factor in human polymorphonuclear neutrophils. Blood 99:2997-3004.

Grenier A, Combaux D, Chastre J, Gougerot-Pocidalo MA, Gibert C, Dehoux M, Chollet-Martin S (2001). Oncostatin M production by blood and alveolar neutrophils during acute lung injury. Lab Invest 81:133-141.

Haslam PL, Turton C, and Heard B (1980). Bronchoalveolar lavage in pulmonary fibrosis: Comparison of cells obtained with lung biopsy and clinical features. Thorax 35:9-18.

Hubbard R, Venn A, Lewis S, and Britton J (2000). Lung cancer and cryptogenic fibrosing alveolitis. Am J Respir Crit Care Med 161:5-8.

Hunninghake GW, Gadek JE, Lawley TJ, and Crystal RG (1981). Mechanisms of neutrophil accumulation in the lungs 
of patients with idiopathic pulmonary fibrosis. J Clin Invest 66:473-483.

Jaffre S, Dehoux M, Paugam C, Grenier A, Chollet-Martin S, Stern J, Mantz J, Aubier M, and Crestani B (2002). Hepatocyte growth factor is produced by neutrophils in the blood and the airspaces in patients with acute respiratory failure. Am J Physiol (Lung Cell Mol Physiol) 282:L310-L315.

Kawanami O, Ferrans VJ, and Crystal RG (1982). Structure of alveolar epithelial cells in patients with fibrotic lung disorders. Lab Invest 46:39-53.

Kim HJ, Sammak PJ, and Ingbar DH (1999). Hepatocyte growth factor stimulates migration of type II alveolar epithelial cells on the provisional matrix proteins fibronectin and fibrinogen. Chest 116 (1 Suppl):94S-95S.

Maslen CL, Hall ND, Woolf AD, and Maddison PJ (1987). Enhanced oxidative metabolism of neutrophils from patients with systemic sclerosis. Br J Rheumatol 26:113-117.

Mason RJ, Leslie CC, McCormick-Shannon K, Deterding RR, Nakamura T, Rubin JS, and Shannon JM (1994). Hepatocyte growth factor is a growth factor for rat alveolar type II cells. Am J Respir Cell Mol Biol 11:561-567.

Matsumoto K, Tajima H, Okazaki H, and Nakamura T (1992). Negative regulation of hepatocyte growth factor gene expression in human lung fibroblasts and leukemic cells by transforming growth factor-beta 1 and glucocorticoids. J Biol Chem 267:24917-24920.

Mine S, Tanaka Y, Suematu M, Aso M, Fujisaki T, Yamada S, and Eto $S$ (1998). Hepatocyte growth factor is a potent trigger of neutrophil adhesion through rapid activation of lymphocyte function-associated antigen-1. Lab Invest 78:13951404.

Moghul A, Lin L, Beedle A, Kanbour-Shakir A, DeFrances MC, Liu Y, and Zarnegar R (1994). Modulation of c-Met proto-oncogene (HGF receptor) mRNA abundance by cytokines and hormones: Evidence of rapid decay of $8 \mathrm{~kb}$ c-Met transcript. Oncogene 9:2045-2052. L L L

Nagahori T, Dohi M, Matsumoto K, Saitoh K, Honda Zl, Nakamura T, and Yamamoto K (1999). Interferon-gamma upregulates the c-Met/hepatocyte growth factor receptor expression in alveolar epithelial cells. Am J Respir Cell Mol Biol 21:490-497.

Ohmichi H, Matsumoto K, and Nakamura T (1996). In vivo mitogenic action of HGF on lung epithelial cells: Pulmotrophic role in lung regeneration. Am J Physiol 270:L1031L1039.

Panos RJ, Patel R, and Bak PM (1996). Intratracheal administration of hepatocyte growth factor/scatter factor stimulates rat alveolar type II cell proliferation in vivo. Am J Respir Cell Mol Biol 15:574-581.

Rennard SI, Basset G, Lecossier D, O’Donnell KM, Pinkston P, Martin PG, and Crystal RG (1986). Estimation of volume of epithelial lining fluid recovered by lavage using urea as marker of dilution. J Appl Physiol 60:532-538.
Sakai T, Satoh K, Matsushima K, Shindo S, Abe S, Abe T, Motomiya M, Kawamoto T, Kawabata Y, Nakamura T, and Nukiwa T (1997). Hepatocyte growth factor in bronchoalveolar lavage fluids and cells in patients with inflammatory chest diseases of the lower respiratory tract: Detection by RIA and in situ hybridization. Am J Respir Cell Mol Biol 16:388-397.

Selman M, King TE, and Pardo A (2001). Idiopathic pulmonary fibrosis: Prevailing and evolving hypotheses about its pathogenesis and implications for therapy. Ann Intern Med 134:136-151.

Stern J-B, Fierobe L, Paugam C, Rolland C, Dehoux M, Petiet A, Dombret M-C, Mantz J, Aubier M, and Crestani B (2000). Keratinocyte growth factor and hepatocyte growth factor in bronchoalveolar lavage fluid in ARDS patients. Crit Care Med 28:2326-2333.

Verghese GM, McCormick-Shannon K, Mason RJ, and Matthay MA (1998). Hepatocyte growth factor and keratinocyte growth factor in the pulmonary edema fluid of patients with acute lung injury: Biologic and clinical significance. Am J Respir Crit Care Med 158:386-394.

Vivekananda J, Awasthi V, Awasthi S, Smith DB, and King RJ (2000). Hepatocyte growth factor is elevated in chronic lung injury and inhibits surfactant metabolism. Am J Physiol (Lung Cell Mol Physiol) 278:L382-L392.

Ware LB and Matthay MA (2002). Keratinocyte and hepatocyte growth factors in the lung: Roles in lung development, inflammation, and repair. Am J Physiol (Lung Cell Mol Physiol) 282:L924-L940.

Weidner KM, Hartmann G, Sachs M, and Birchmeier W (1993). Properties and functions of scatter factor/hepatocyte growth factor and its receptor c-met. Am J Respir Cell Mol Biol 8:229-237.

Yaekashiwa M,-Nakayama S, Ohnuma K, Sakai T, Abe T, Satoh K, Matsumoto K, Nakamura T, Takahashi T, and Nukiwa T (1997). Simultaneous or delayed administration of hepatocyte growth factor equally represses the fibrotic changes in murine lung injury by bleomycin: A morphologic study. Am J Respir Crit Care Med 156:1937-1944.

Yamanouchi H, Fujita J, Yoshinouchi T, Hojo S, Kamei T, Yamadori I, Ohtsuki Y, Ueda N, and Takahara J (1998). Measurement of hepatocyte growth factor in serum and bronchoalveolar lavage fluid in patients with pulmonary fibrosis. Respir Med 92:273-278.

Yamashita J, Ogawa M, Nakano S, Okabe K, Abe M, Iwasaki A, Kuwahara M, Yoshinaga Y, and Shirakusa T (1998). High levels of hepatocyte growth factor/scatter factor in diffusetype bronchioloalveolar cell carcinoma. Cancer 83:20912098.

Yanagita K, Matsumoto K, Sekiguchi K, Ishibashi H, Niho Y, and Nakamura T (1993). Hepatocyte growth factor may act as a pulmotrophic factor on lung regeneration after acute lung injury. J Biol Chem 268:21212-21217. 